\title{
Hydrogen Analysis by Ultra-High Energy Resolution EELS
}

H. Cohen ${ }^{1}$, P. Rez ${ }^{2}$, T. Aoki ${ }^{3}$, P.A. Crozier ${ }^{3,4}$, N. Dellby ${ }^{5}$, Z. Dellby ${ }^{5}$, D. Gur ${ }^{6}$, T.C. Lovejoy ${ }^{5}$,

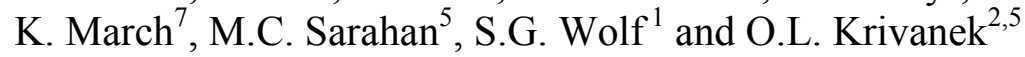

1. Department of Chemical Research Support, Weizmann Institute of Science, Rehovot 76100, Israel

2. Department of Physics, ASU, Tempe, AZ 85287, USA

3. Center for Solid State Science, Arizona State University, Tempe, AZ 85287, USA

4. School for Engineering of Matter, Transport and Energy, ASU, Tempe, AZ 85287, USA

5. Nion Co., 11511 NE $118^{\text {th }}$ St., Kirkland, WA 98034, USA

6. Structural Biology, Weizmann Institute of Science, Rehovot 76100, Israel

7. Physique des Solides, University of Paris-Sud, Orsay 91405, France

Hydrogen is a difficult element to detect by electron microscopy (EM). Being very light, its elastic scattering cross-section is much smaller than for other elements. Even worse, it does not give rise to any core loss edges that can be used for detecting it by electron energy loss spectroscopy (EELS), nor to the emission of characteristic X-rays or Auger electrons. In metals, hydrogen typically becomes a loosely bound proton, plus an electron that joins the valence band. In organic materials it is bound more tightly, but it is easily ejected by knock-on or ionization damage. Its detection has been reported by annular bright field (ABF) STEM [1] and other methods such as measuring plasmon energy shifts, but not by a general method applicable to all materials. A reliable EM spectroscopic method for mapping hydrogen would be very useful, especially if it were also able to provide information on its bonding.

Ultra-high energy resolution (UHR) EELS is now able to provide such a method. A new type of an electron monochromator [2] has improved the EELS energy resolution about 3-fold, to $\sim 10 \mathrm{meV}$ at 60 $\mathrm{keV}$ primary energy, and this has made vibrational spectroscopy possible in the electron microscope [3]. With its single proton nucleus, hydrogen gives the highest energy vibrational peaks and is therefore the easiest element to detect by the vibrational approach, in principle. Fig. 1 shows vibrational spectra of titanium hydride $\left(\mathrm{TiH}_{2}\right)$ and epoxy resin [3]. The vibrational peak due to the loosely bound hydrogen in $\mathrm{TiH}_{2}$ is at $147 \mathrm{meV}$ and thus lies on a large background due to the zero loss peak (ZLP), but it can be readily extracted using a power-law fit. The main vibrational peak in the epoxy resin is due to more strongly bound hydrogen and occurs at $360 \mathrm{meV}$. The cross-sections are of the order of $1 \mathrm{pm}^{2}$ per atom, i.e. similar to the carbon $\mathrm{K}$ shell ionization cross section [4]. However, because the vibrational signal is concentrated into narrow peaks rather than an edge tens of $\mathrm{eV}$ wide, it is more readily detectable.

Due to the low energies, a large part of the vibrational signal is "delocalized" and can be excited even with an "aloof" electron beam positioned tens of $\mathrm{nm}$ in the vacuum next to the sample [3]. $\mathrm{The} \mathrm{TiH}_{2}$ spectrum was in fact acquired with an aloof beam of $\sim 2 \mathrm{~nm}$ diameter stationed $\sim 30 \mathrm{~nm}$ in the vacuum outside a $\mathrm{TiH}_{2}$ particle. Provided that the beam is substantially tail-free, radiation damage is then greatly reduced, as the only transitions the aloof beam can excite in the sample occur at energies $<1 \mathrm{eV}$. This means that if the sample can tolerate infrared light, it will not be greatly damaged by the aloof beam.

For the epoxy spectrum, the beam was defocused and covered a sample patch of $\sim 400 \mathrm{~nm}$ diameter [3], to minimize radiation damage per unit area. A disadvantage of this approach is that the wide beam is sensitive to sample charging which can act as an imperfect "lens" that adds aberrations worsening the spectrum quality. A more robust approach for minimizing radiation damage is to scan a focused beam rapidly over the sample and de-scan it after the sample, and we are implementing this mode. 
The best signal-to-noise ratio in vibrational spectra of beam-sensitive materials is usually obtained in the aloof mode, albeit at a reduced spatial resolution. Fig. 2 shows a UHR EEL spectrum of guanine acquired at $60 \mathrm{keV}$, with the beam parked $60 \mathrm{~nm}$ outside the sample. The agreement with the infrared spectrum is excellent, although the EELS energy resolution $(\sim 16 \mathrm{meV})$ is considerably worse. As is typical of vibrational spectroscopies, the different peaks can be assigned to different types of bonds and vibration modes (see the inset in Fig. 2). This opens up many interesting possibilities, including being able to monitor how different types of hydrogen bonds stand up to electron exposure.

A further improvement of energy resolution by about $2 \mathrm{x}$ should be straightforward with our approach, provided that both the monochromator and the spectrometer are brought up to the rigorous stability standards developed for aberration-corrected microscopes. The spatial resolution of the analysis can most likely also be improved substantially, by focusing on high-angle EELS scattering events [5]. To summarize this exciting new development, UHR EELS promises to make the analysis of hydrogen as routine and potentially more informative than analytical electron microscopy of any other element.

References:

[1] R. Ishikawa et al., Nature Materials 10 (2011) 278-281.

[2] O.L. Krivanek et al., Microscopy 62 (2013) 3-21.

[3] O.L. Krivanek et al., Nature 514 (2014) 209-212.

[4] P. Rez, Microsc. Micoanal. 20 (2014) 671-677.

[5] T.C. Lovejoy et al., Microsc. Micoanal. 20 suppl. 3 (2014)558-559.

[6] We gratefully acknowledge the use of facilities within the LeRoy Eyring Center for Solid State Science at ASU, and grants NSF MRI-R2 \#959905 and DE-SC0004954 and DE-SC0007694.

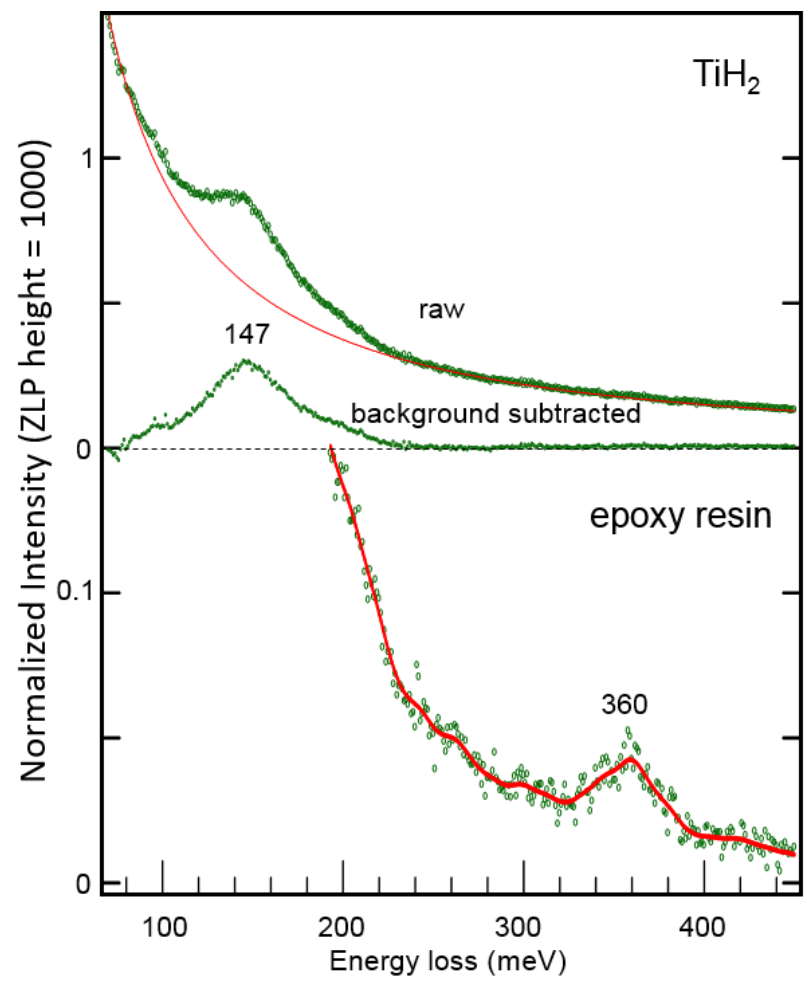

Figure 1. UHR EEL spectra of $\mathrm{TiH}_{2}$ and epoxy resin. Nion UltraSTEM100MC, $60 \mathrm{kV}$.

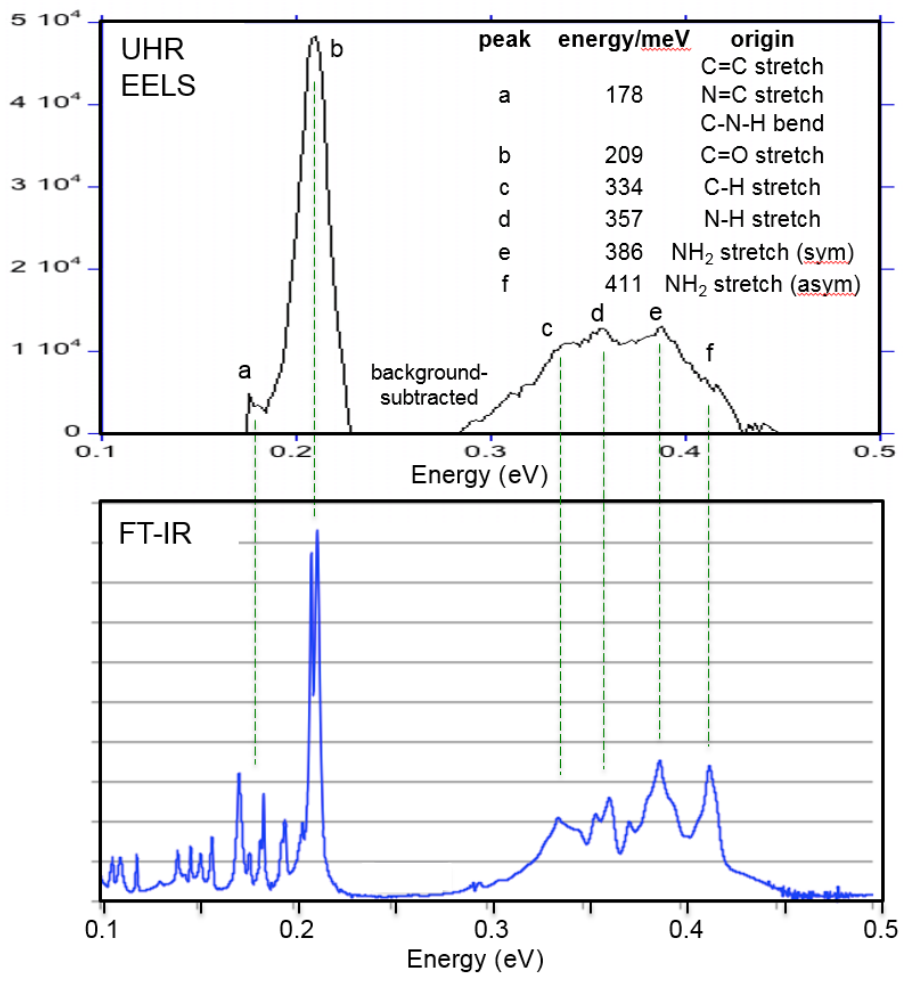

Figure 2. Top: UHR EEL spectrum of guanine (Nion UltraSTEM100MC, $60 \mathrm{kV}$ ), aloof mode. Bottom: infrared spectrum of guanine. 